\title{
Splenic Abscess
}

National Cancer Institute

\section{Source}

National Cancer Institute. Splenic Abscess. NCI Thesaurus. Code C35347.

An abscess that is located in the spleen. 